\section{TICKER}

Chirurgie: „Montagspatienten“ haben die besten Überlebenschancen

Ein Patient, der statt an einem Montag an einem Freitag operiert wird, hat ein 1,44mal höheres postoperatives Sterberisiko. Die Mortalität nach Eingriffen am Wochenende liegt sogar fast doppelt so hoch. Dies zeigt eine britische Studie, die die 30-Tages-Sterblichkeit von Patienten verglichen hat, die an verschiedenen Wochentagen operiert wurden.

E Unter den 4133346 Patienten starben 27582 innerhalb von 30 Tagen nach dem elektiven Eingriff. Die Sterberaten waren umso höher, je näher der Op.-Tag Richtung Wochenende rückte. Verglichen mit Patienten, die an einem Montag operiert wurden, hatten Patienten mit Freitags-Eingriffen eine um 44\% höhere Wahrscheinlichkeit, die kommenden 30 Tage nicht zu überleben. Fand eine Operation am Samstag oder Sonntag statt, stieg das Risiko sogar um $82 \%$. ST = Aylin P et al. BMJ 2013; 346: f2424, online 28. Mai

Jeder dritte Deutsche ist Hypertoniker Rund 30\% der erwachsenen Frauen und 33\% der Männer in Deutschland leiden unter Hypertonie. Von den 70- bis 79-Jährigen stehen drei Viertel unter zu hohem Druck. Immerhin, so ein Ergebnis der „Studie zur Gesundheit Erwachsener in Deutschland (DEGS)", wissen mehr als 80\% der Hypertoniker offenbar von ihrer Krankheit. In der DEGS wurde bei 7096 18- bis 79-jährigen Teilnehmern standardisiert der Blutdruck ermittelt.

Bei Frauen lag der mittlere Blutdruck systolisch bei $120,8 \mathrm{mmHg}$ und diastolisch bei 71,2 mmHg. Die Mittelwerte der Männer betrugen 127,4 mmHg bzw. 75,3 $\mathrm{mmHg}$. Unter den 18- bis 29-Jährigen hatten $8 \%$ der Männer hypertone und $33 \%$ optimale Werte. Dagegen wurden bei nur 1\% der jungen Frauen zu hohe Werte gemessen, bei $76 \%$ war der Blutdruck optimal.

Neuhauser Het al. Bundesgesundheitsbl 2013; 56: 795-801

\title{
Nüsse und Olivenöl bringen das Gehirn auf Trab
}

- Besser das richtige Fett als gar kein Fett - für die Hirnleistung von Älteren scheint eine mediterrane Diät mit Nüssen und/oder Olivenöl besser zu sein als eine fettreduzierte Kost, berichten spanische Forscher. An ihrer sog. PREDIMED-Studie nahmen über 1000 Personen teil, die in drei verschiedene Gruppen aufgeteilt wurden: Sie sollten sich entweder nur fettarm ernähren, mediterran mit Olivenöl oder mediterran mit Nüssen. Die Olivenölgruppe bekam dazu jede Woche einen Liter Olivenöl zugeteilt, die Nussgruppe erhielt pro Tag $15 \mathrm{~g}$ Walnüsse, 7,5 g Mandeln und 7,5 g Haselnüsse.

\section{Ein halber Punkt Abweichung beim Mini-Mental-Status-Test}

Die Forscher präsentierten nun eine Zwischenauswertung mit Daten von etwa der Hälfte der Teilnehmer. Darunter befanden sich 266 Personen aus der Olivenöl-, 166 aus der Nuss- und 132 aus der Kontrollgruppe. Die Teilnehmer waren zum Studienende im Schnitt etwa 74 Jahre alt und unterzogen sich dann einer neuropsychologischen Untersuchung, unter anderem mit dem Mini-Mental-Status-Test (MMST) und dem Uhrentest.
Die Ergebnisse: Der MMST-Wert lag in den drei Gruppen zum Ende zwischen 27 und knapp über 28 Punkten, der Wert beim Uhrentest bei 5,0-5,5 Punkten. Die Teilnehmer der Olivenölgruppe erreichten den höchsten MMST-Wert, er lag im Schnitt 0,6 Punkte über dem der Kontrollgruppe mit der fettarmen Kost. 0,57 Punkte betrug die Abweichung zwischen Nuss- und Kontrollgruppe - die Unterschiede waren jeweils signifikant. Ebenfalls signifikant waren die Unterschiede beim Uhrentest: 0,51 Punkte mehr in der Olivenöl- und 0,33 Punkte mehr in der Nussgruppe als in der Kontrollgruppe. Die Werte wurden u. a. unter Berücksichtigung von Bildungsgrad, BMl, Alkoholkonsum und Energiebedarf berechnet. Der Anteil der Teilnehmer mit ersten kognitiven Beeinträchtigungen war in der Olivenölgruppe am geringsten (8\%), gefolgt von Nuss- (11\%) und Kontrollgruppe (17\%).

Die Autoren sehen die Studie als Hinweis, dass eine mediterrane Diät mit Nüssen und Olivenöl die Kognition im Alter unterstützt. Als Mechanismen werden antioxidative Eigenschaften von Öl und Nüssen diskutiert.

MUT -

- Martinez-Gonzalez MA et al. J Neurol Neurosurg Psych 2013; DOI: 10.1136/jnnp-2013-305153.

\section{PATIENTENGLAUBE VS. STUDIENLAGE}

\section{Wie wetterfühlig ist die Fibromyalgie?}

\section{- Viele Fibromyalgiepati-} enten berichten, dass ihre Beschwerden wetterabhängig sind. Niederländische Wissenschaftler haben die Probe gemacht. Hierzu ließen sie 333 Fibromyalgiepatientinnen 28 Tage lang ein Symptomtagebuch führen und setzten das Ergebnis zu den lokalen Wet-

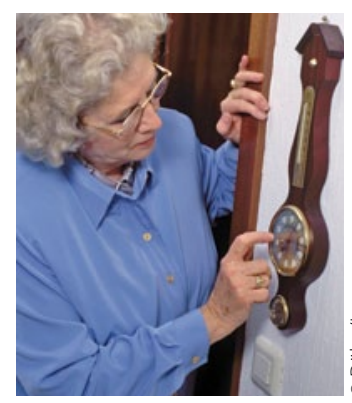

rigen oder gleichen Tag), jeweils für Schmerz und für Müdigkeit. Von den insgesamt 50 Analysen zeigten aber nur fünf eine signifikante, wenn auch schwache ơ Korrelation zwischen Wetter und Symptom. So gingen eine Zunahme der Sonnenscheindauer mit einer $\mathrm{Ab}$ - teraufzeichnungen in Beziehung. Analysiert wurden fünf Wettervariablen (Temperatur, Sonnenscheindauer, Niederschlag, Luftdruck und Luftfeuchtigkeit) und fünf Arten von Assoziationen (bestimmte Wetterbedingungen oder ein Wetterwechsel am vo- schwächung und ein Anstieg der Luftfeuchtigkeit mit einer Verstärkung der Schmerzen einher. Müdigkeit war positiv korreliert mit der Temperatur.

BS = accepted article; doi: 10.1002/acr.22008 